\title{
BMJ Open Physical activity, immune function and inflammation in kidney patients (the PINK study): a feasibility trial protocol
}

Patrick James Highton, ${ }^{1,2}$ Jill Neale, ${ }^{2}$ Thomas J Wilkinson, ${ }^{2}$ Nicolette C Bishop, ${ }^{1,2}$ Alice C Smith ${ }^{1,2}$

To cite: Highton PJ, Neale J, Wilkinson TJ, et al. Physical activity, immune function and inflammation in kidney patients (the PINK study): a feasibility trial protocol. BMJ Open 2017;7:e014713. doi:10.1136/ bmjopen-2016-014713

- Prepublication history and additional material are available. To view these files please visit the journal online (http://dx.doi.org/ 10.1136/ bmjopen-2016-014713)

Received 21 October 2016 Revised 21 March 2017 Accepted 28 March 2017

\section{CrossMark}

${ }^{1}$ School of Sport, Exercise and Health Sciences, Loughborough University, Loughborough, UK ${ }^{2}$ Department of Infection, Immunity and Inflammation, University of Leicester, Leicester, UK

Correspondence to Patrick James Highton; p.highton@lboro.ac.uk

\section{ABSTRACT}

Introduction Patients with chronic kidney disease (CKD) display increased infection-related mortality and elevated cardiovascular risk only partly attributed to traditional risk factors. Patients with CKD also exhibit a pro-inflammatory environment and impaired immune function. Aerobic exercise has the potential to positively impact these detriments, but is under-researched in this patient population. This feasibility study will investigate the effects of acute aerobic exercise on inflammation and immune function in patients with CKD to inform the design of larger studies intended to ultimately influence current exercise recommendations.

Methods and analysis Patients with CKD, including renal transplant recipients, will visit the laboratory on two occasions, both preceded by appropriate exercise, alcohol and caffeine restrictions. On visit 1, baseline assessments will be completed, comprising anthropometrics, body composition, cardiovascular function and fatigue and leisure time exercise questionnaires. Participants will then undertake an incremental shuttle walk test to estimate predicted peak $\mathrm{O}_{2}$ consumption ( $\mathrm{VO}_{2}$ peak). On visit 2 , participants will complete a 20 min shuttle walk at a constant speed to achieve $85 \%$ estimated V0 2 peak. Blood and saliva samples will be taken before, immediately after and 1 hour after this exercise bout. Muscle $\mathrm{O}_{2}$ saturation will be monitored throughout exercise and recovery. Age and sex-matched non-CKD 'healthy control' participants will complete an identical protocol. Blood and saliva samples will be analysed for markers of inflammation and immune function, using cytometric bead array and flow cytometry techniques. Appropriate statistical tests will be used to analyse the data.

Ethics and dissemination A favourable opinion was granted by the East Midlands-Derby Research Ethics Committee on 18 September 2015 (ref 15/EM/0391), and the study was approved and sponsored by University Hospitals of Leicester Research and Innovation (ref 11444). The study was registered with ISRCTN (ref 38935454). The results will be presented at relevant conferences, and it is anticipated that the reports will be published in appropriate journals in 2018.

\section{INTRODUCTION}

Patients with all stages of chronic kidney disease (CKD) have elevated cardiovascular disease (CVD) risk that cannot be fully
Strengths and limitations of this study

Inclusion of control group matched for age and sex.

- A variety of outcome measures to inform future study design.

- Pragmatic study design which will inform future exercise recommendations for patients with CKD.

- No non-exercise control visit.

explained by traditional risk factors. ${ }^{1}$ CVD is the most common cause of death among patients with CKD (23\% in 2013), followed by infection (19\% in 2013). ${ }^{2}$

Patients with CKD are often sarcopenic and obese,,$^{3-6}$ and further deterioration in these characteristics is often observed after renal transplantation. ${ }^{7-9}$ Patients with CKD and renal transplant recipients (RTRs) also frequently suffer from chronic systemic inflammation, ${ }^{10} 11$ which can worsen cachexia and increase cardiovascular risk in CKD. ${ }^{12}{ }^{13}$ Patients with CKD and RTRs also display impaired cellular immune function, ${ }^{14-16}$ which may explain why infection is the second-leading cause of death in this population. ${ }^{2}$ This effect (ie, impaired immune function) is further compounded in RTRs by the immunosuppressive drug regime. Impaired immune function may further exacerbate inflammation due to alterations in circulating immune cell subsets ${ }^{17}$ which could serve to worsen body composition and increase cardiovascular risk. Finally, patients with CKD display elevated levels of circulating prothrombotic microparticles (MPs) ${ }^{18}$ which may worsen CVD risk and/or burden. ${ }^{19}$

Exercise has the potential to benefit and improve many of the aforementioned inter-related morbidities. In the general population, exercise can positively impact on weight gain, ${ }^{20}$ muscle wasting, ${ }^{21}$ physical capacity ${ }^{22}$ and fatigue. ${ }^{23}$ Furthermore, moderate aerobic exercise can also modify systemic inflammation, ${ }^{24}{ }^{25}$ bolster immune 
function via alterations in circulating immune cell populations and activity and reduce circulating MP levels. ${ }^{26-29}$

In the CKD population, similar positive effects of exercise on body composition, physical function and quality of life have been demonstrated ${ }^{30-33}$; however, the research on inflammation and immune function in this population is limited. Previous research has shown that 30 min of moderate intensity walking exercise elicited a normal pattern of leucocyte and monocyte activation, while enhancing granulocyte function and promoting an anti-inflammatory environment (increased IL-10 release) in patients with pre-dialysis CKD. ${ }^{34}$ However, in a similar population, an exhaustive bout of cycling exercise elicited a shift towards the more proinflammatory $\mathrm{CD} 16^{+}$ monocyte, potentially favouring inflammation. ${ }^{35}$ This disparity may be due to the exercise intensities investigated-moderate intensity exercise is more beneficial in strengthening immune function and preventing infection $^{26}$ and is promoted in general exercise guidelines. As such, investigating the effects of moderate intensity exercise in this patient population is more pragmatic and will help to guide future exercise recommendations, which are lacking in this patient population.

Therefore, this feasibility study aims to investigate the effects of acute aerobic walking exercise on the inflammation and immune function in patients with CKD. This will create the basis from which a larger trial can be conducted, including data for a power calculation, which will ultimately help to inform exercise recommendations in this population which are currently lacking, particularly with regards to immune function and inflammation. ${ }^{36}$ However, a preliminary power calculation (GPower V.3.1) based on the findings of Viana et $a l^{34}$ concerning total lymphocyte count (based on a statistical power of 0.80 and an alpha level of 0.05 ) suggests that 15 participants per group (eg, predialysis CKD, RTRs and healthy controls) will be sufficient.

\section{METHODS AND ANALYSIS}

\section{Outcomes to be measured}

This study will investigate the effect of 20 min of moderate intensity walking exercise on immune cell subsets and inflammatory markers. Participants will be grouped based on their status (ie, 'patient' or 'healthy control'). Both groups will complete an identical acute, cross-sectional study protocol necessitating two study visits, as explained below.

\section{Participant recruitment}

All patients with CKD attending outpatient clinics within the University Hospitals of Leicester renal network will be screened for eligibility by their consultant nephrologist prior to recruitment, and approached during their routine outpatient appointments. The inclusion and exclusion criteria for patients are summarised in table 1 . Healthy age-matched and sex-matched controls with no known chronic disease will be recruited from the local community. Those who do not believe themselves to suffer from any significant chronic disease will be eligible to participate. The 'broad' inclusion and exclusion criteria allow the incorporation of several different groups (ie, patients with non-dialysis CKD, with dialysis, RTRs and healthy controls), and thus will allow comparison between these groups. Similarly, the wide range of CKD stage will allow the influence of remaining renal function on the measured markers to be investigated.

\section{Trial design and timeline}

This is a non-randomised, controlled, feasibility study. Participants will complete two study visits as described below.

\section{Visit 1}

Participants will arrive at the laboratory in the morning, unfasted but having consumed no caffeine or alcohol and completed no strenuous exercise for 24 hours. Participants will complete questionnaires about time spent in leisure activity and their perception of fatigue, and assessments of anthropometry, body composition and cardiovascular condition. Participants will then undertake the Incremental Shuttle Walk Test (ISWT), followed by the Endurance Shuttle Walk Test (ESWT) as explained below. If the participant cannot complete the full 20 min duration of the ESWT, they will be withdrawn from the study.

\section{Visit 2}

Participants will arrive at the laboratory in the morning (8-10 am, to minimise the influence of diurnal variation

\begin{tabular}{ll}
\hline Table 1 Inclusion and exclusion criteria for patients \\
\hline Inclusion criteria & Exclusion criteria \\
\hline $\begin{array}{l}\text { Established chronic kidney } \\
\text { disease (all stages will be }\end{array}$ & Age under 18years \\
eligible including those & Pregnancy \\
with an established kidney & Received kidney transplant less than 6 months prior to study entry \\
transplant and those & Any element of study assessment protocol considered by principle care provider to be \\
receiving dialysis treatment) & contraindicated due to physical impairment, comorbidity or any other reason \\
& Inability to give informed consent for any reason \\
& Visual or hearing impairment or insufficient command of English to give informed consent \\
& or comply with the assessment protocol
\end{tabular}




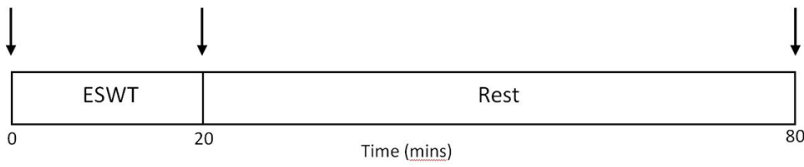

Figure 1 Visit 2 exercise and sample collection protocol. $\downarrow$, venous blood and saliva collection. ESWT, Endurance Shuttle Walk Test.

on immune parameters), following the same standardisation procedure as in visit 1 . After resting for $10 \mathrm{~min}$, blood and saliva samples will be collected. The ESWT will then be completed at the same speed as in visit 1 , lasting for $20 \mathrm{~min}$. Within $5 \mathrm{~min}$ of exercise cessation, another blood and saliva sample will be collected. The participant will then rest for 1 hour, after which a final blood and saliva samples will be collected. This protocol is summarised in a schematic in figure 1, and the outcome measures are explained in greater detail below.

The time delay between recruitment, visit land visit 2 will be kept to a minimum to prevent deconditioning and minimise dropout rates. For dialysis patients, both study visits will be completed on a non-dialysis day that is not after their 'long break' (ie, weekend or two consecutive days without dialysis based on their regular shift pattern) to minimise the effect of fluid overload.

\section{Physical performance}

Endurance capacity will be assessed using the ISWT ${ }^{37}$ and ESWT. ${ }^{38}$ In the ISWT, the participant walks a level $10 \mathrm{~m}$ shuttle course at a speed controlled by an external audible bleep signal. The test progressively increases at 1 min interval for a total of 12 intervals and is terminated when the participant fails to complete a shuttle within the required time. Following the ISWT, a walking speed equating to $85 \%$ of predicted peak $\mathrm{O}_{2}$ consumption $\left(\mathrm{VO}_{2}\right.$ peak) can be calculated, using a conversion table based on their ISWT performance. The ESWT is completed at this continuous speed on the same shuttle course until volitional exhaustion or the end of the test $(20 \mathrm{~min})$ is reached. For the purposes of this study, any participant who does not complete the full $20 \mathrm{~min}$ of the ESWT in visit one will be excluded to ensure standardisation of the test in visit 2. This protocol was initially developed for use in patients with chronic airways obstruction ${ }^{37}$ but has been used in the CKD population, ${ }^{39} 40$ showing good reproducibility and a high correlation with $\mathrm{VO}_{2}$ peak. ${ }^{41}$

\section{Venous blood sampling}

Venous blood will be collected using venepuncture of the antecubital vein of either arm-provided the absence of an arteriovenous fistula. Blood will be drawn through a 21- gauge needle $(30 \mathrm{~mL}$ per time point) and collected into di-potassium EDTA, sodium citrate, sodium heparin and blank monovettes.

\section{Blood processing, storage and analysis}

Blood collected into EDTA tubes will be centrifuged at $4^{\circ} \mathrm{C}$; the supernatant will then be aliquoted and frozen at $-80^{\circ} \mathrm{C}$ for future analysis. A cytometric bead array technique will be used to allow bulk analysis of a panel of proinflammatory and anti-inflammatory cytokines, including but not limited to IL-1, IL-2, IL-6, IL-10, tumour necrosis factor alpha and interferon gamma. Blood collected into sodium citrate ${ }^{42}$ will be double centrifuged at room temperature $(15 \mathrm{~min}$ at $2500 \mathrm{~g}$, supernatant aliquoted followed by another 15 min at $2500 \mathrm{~g}$ ) to create platelet-poor plasma; the supernatant will then be aliquoted and frozen at $-80^{\circ} \mathrm{C}$ for future phenotyping of microparticles using flow cytometry, as explained below. Blood collected into sodium heparin will be analysed on the day of collection for immune cell subsets using flow cytometry, as explained below. Blood collected into blank monovettes will be sent to the Diagnostic Pathology Service at University Hospitals of Leicester National Health Service (NHS) Trust on the day of collection for renal profile analysis, which includes estimated glomerular filtration rate, urea, bicarbonate, creatine, sodium, potassium and phosphate measures. This will be completed for both patient and control populations.

\section{Flow cytometry}

Immune cells will be characterised based on their expression of surface antigens using flow cytometry. Monocyte subsets will be categorised as classical $\left(\mathrm{CD} 14^{++} \mathrm{CD} 16^{-}\right)$, intermediate $\left(\mathrm{CD} 14^{++} \mathrm{CD} 16^{+}\right)$and non-classical $\left(\mathrm{CD} 14^{+} \mathrm{CD} 16^{++}\right) \cdot{ }^{43}$ Monocyte angiotensin-converting enzyme expression will be assessed using CD143, with immunoglobulin G1 as a negative control. $\mathrm{T}$ cells will be categorised as helper $\left(\mathrm{CD}^{+} \mathrm{CD}^{+}\right)$cytotoxic $\left(\mathrm{CD}^{+} \mathrm{CD}^{+}\right)$and regulatory $\left(\mathrm{CD} 4^{+} \mathrm{CD} 25^{+} \mathrm{CD} 127^{-}\right)$ $\mathrm{T}$ cells. B Cells will be identified as $\mathrm{CD}^{+} \mathrm{CD} 19^{+}$and NK Cells as CD3-CD56 ${ }^{+}$. Following appropriate staining and washing procedures, immune cells will be analysed on an FACSCalibur (BD Biosciences, Oxford, UK). Acquisition templates and preliminary flow cytometry results are displayed in figure 2.

Microparticles will be characterised based on their size, Annexin-v expression, cellular derivation and prothrombotic potential. Cellular derivations will be categorised as platelet derived $\left(\mathrm{CD} 42 \mathrm{~b}^{+}\right)$, neutrophil derived $\left(\mathrm{CD} 66 \mathrm{~b}^{+}\right)$, monocyte derived $\left(\mathrm{CD} 14^{+}\right)$and endothelial cell derived $\left(\mathrm{CD} 144^{+}\right)$. Prothrombotic potential will be estimated using Tissue Factor $\left(\mathrm{CD} 142^{+}\right)$expression. Following the thawing of platelet-free plasma at room temperature, samples will be double centrifuged at room temperature (30 min at $18000 \mathrm{~g}$, supernatant removed, discarded and replaced with an equal volume of buffer, followed by another $30 \mathrm{~min}$ at $18000 \mathrm{~g}$ ). Samples will then undergo appropriate staining procedures with the antibodies listed above, after which microparticles will be analysed on a BD Accuri C6 (BD Biosciences, Oxford, UK) flow cytometer. Acquisition templates and preliminary microparticle 

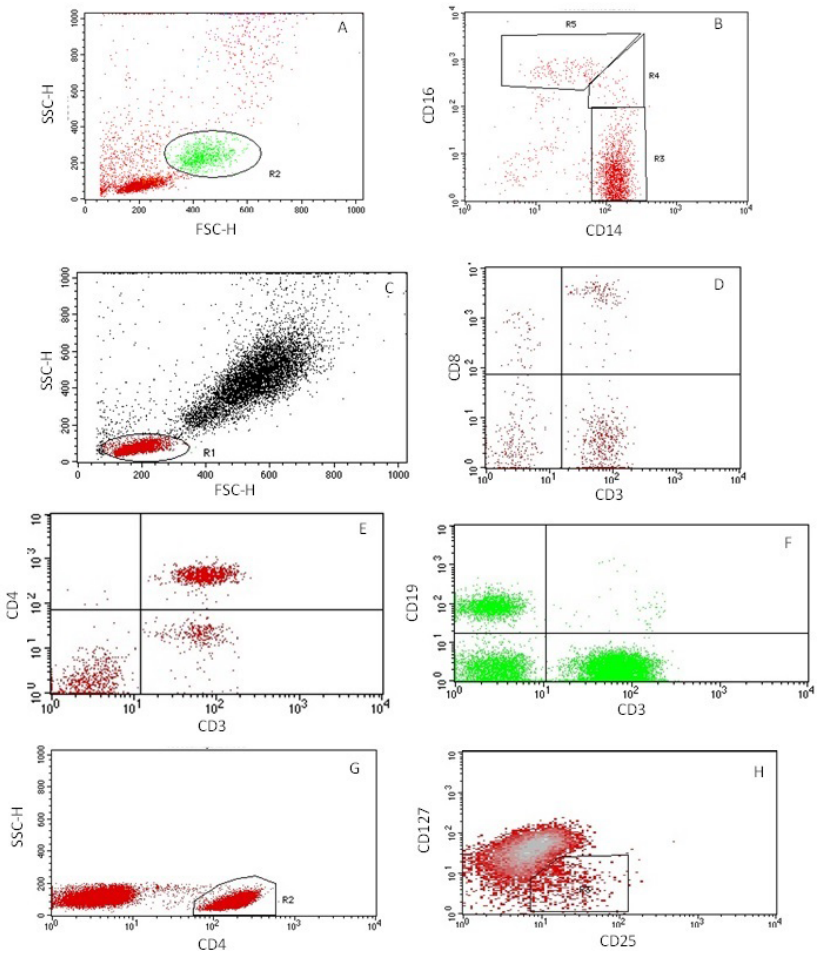

Figure 2 Demonstration of the gating strategy used to identify immune cell subsets. (A) Total monocyte gating. (B) Monocyte subsets (R3 = classical (CD14++CD16-), R4 = intermediate (CD14++CD16+), R5 = non-classical (CD14+CD16++) (R1 and R2 were used as preliminary gating to remove neutrophils). (C) Total lymphocyte gating. (D) Gating CD8+ cytotoxic T lymphocytes in the upper right quadrant. (E) Gating CD4+ helper T lymphocytes in the upper right quadrant. (F) Gating B lymphocytes (CD3-CD19+) in the upper left quadrant. (G) Initial T-Reg gating, identifying CD4+ lymphocytes. $(H)$ Secondary T-reg gating, back-gated onto plot G, further identifying the CD4+ lymphocytes that are CD25+CD127-. Not all graphs display $100 \%$ of acquired cells - this has been altered independently to allow ease of gating.

results are displayed in figure 3. The protocol for isolating and analysing MPs is based on a number of publications $^{42} 4445$ and in our experience produces reliable results (figure 3 ).

\section{Saliva sampling and storage}

Saliva samples will be collected into sterile plastic containers. Participants will swallow to empty the mouth, then open and hold the container themselves before performing a passive dribble of saliva collected under the tongue over the next $2 \mathrm{~min}$. Following centrifugation, the supernatant will be aliquoted and frozen for future analysis, primarily for secretory IgA to investigate mucosal immunity.

\section{Anthropometric measures}

Height, weight and waist and hip circumference will be measured using standard procedures. ${ }^{46}$
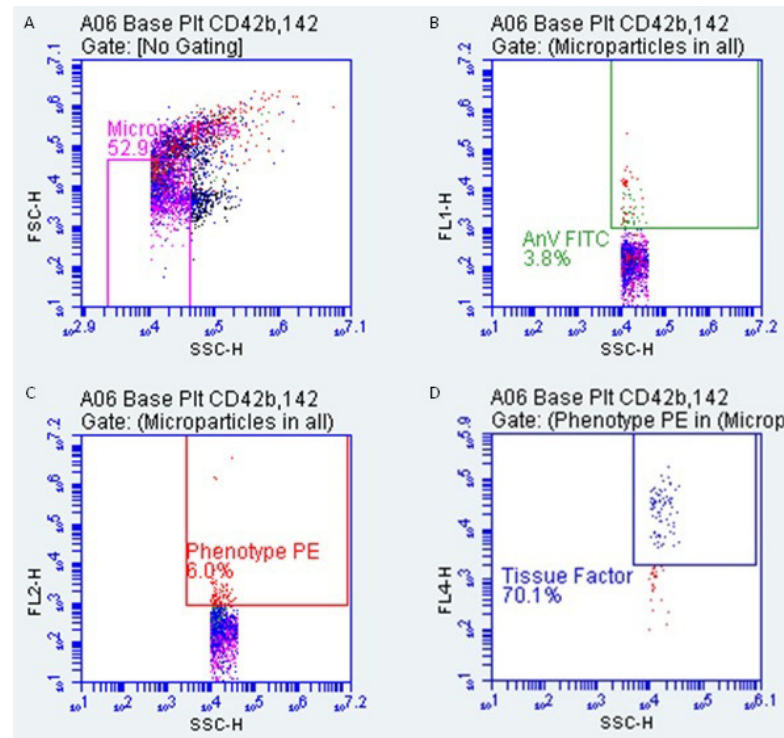

D A06 Base PIt CD 42b,142 Gate: (Phenotype PE' in (Microparticl

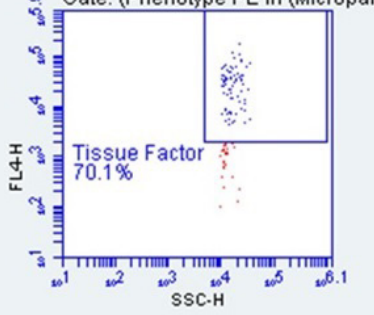

Figure 3 Demonstration of the gating strategy used to characterise microparticles. (A) 'All microparticles' set using beads of known size. (B) 'All microparticles' based on Annexin-v expression. (C) Phenotype marker expression, used to quantify microparticles of different cellular sources. (D) Prothrombotic potential, assessed by Tissue Factor expression. All gates have been appropriately sized and positioned using unstained samples to distinguish negative versus positive staining. FITC, fluorescein isothiocyanate; F/ SSC-H, Forward/Side Scatter-Height; PE phycoerythrin.

\section{Body composition}

Body composition parameters (ie, fat and fat-free mass content) will be measured using bioelectrical impedance analysis (BIA) (Inbody 370, Chicago, Illinois, USA). BIA has been validated for use in CKD. ${ }^{47}$ While BIA has limitations with regards to reliability and is not recommended for tracking changes in body composition, body composition will only be used as a descriptive rather than an outcome variable, and thus BIA has been deemed suitable to assess body composition for this reason.

\section{Cardiovascular condition and function}

Cardiac bioreactance analysis will be carried out using a Non-Invasive Cardiac Output Monitor (Cheetah Medical, Maidenhead, UK). Cardiac bioreactance is a quick, safe, validated $^{48}$ and non-invasive method of assessing central haemodynamics including heart rate, stroke volume, cardiac output and total peripheral resistance. This is accomplished by using cutaneous electrodes placed on the chest in combination with an automatic sphygmomanometer to measure blood pressure.

\section{Time spent in leisure activities}

The Leisure Time Exercise Questionnaire assesses the amount of exercise a patient undertakes, from which a metabolic equivalent estimation can be calculated. It also identifies preferred physical activities. 


\section{Fatigue}

Fatigue is a common complaint CKD, and it has multiple potential mechanisms including anaemia ${ }^{49}$ and cachexia. ${ }^{50}$ To assess self-reported fatigue, participants will be asked to fill in a fatigue scale (an internally designed Likert scale see online supplementary material), as well as the validated Functional Assessment of Chronic Illness Therapy-Fatigue $^{51}$ which has been extensively used in CKD populations ${ }^{52}$

\section{Muscle $\mathrm{O}_{2}$ saturation}

Reduced muscle $\mathrm{O}_{2}$ saturation is another possible physiological mechanism of fatigue $\mathrm{e}^{53}$ and may be related to increased inflammation. ${ }^{54} \mathrm{~A}$ small non-invasive muscle $\mathrm{O}_{2}$ saturation device (BSXInsight, BSXAthletics, Texas, USA) will be fitted around the participant's calf and worn during exercise bouts and subsequent recovery. This device uses near infrared spectroscopy (NIRS) to measure haemoglobin and myoglobin oxygenation (muscle $\mathrm{O}_{2}$ tissue stores). NIRS has previously been used in a variety of disease conditions. ${ }^{55}$

\section{Clinical information}

Clinical records will be used to extract information that allows associations to be made with physical activity levels and account for confounding variables, including: age, gender, ethnicity, primary cause of renal failure, transplant or dialysis type, time since transplant or duration of dialysis, comorbidities and current medications.

\section{Data analysis plan}

Mixed-design analyses of variance will be used to analyse main effects of group and time and interaction effects, with Bonferroni post hoc testing used to elucidate these effects. This will generate both tests of significance and estimates of effect sizes. Any non-normally distributed data will first be transformed appropriately to ensure normality assumptions are met, to increase statistical power and to allow clear conclusions to be drawn from the data. Where normality assumptions cannot be met via data transformation, Friedman's tests will be employed as a non-parametric alternative. This will allow the generation of hypotheses for future, larger studies.

\section{Ethics and dissemination}

\section{Ethical and safety considerations}

This protocol was reviewed by the East MidlandsDerby Research Ethics Committee (REC) and was given a favourable opinion (REC ref 15/EM/0391) on 24 September 2015. Additionally, local approval was given by the Research and Innovation office at the University Hospitals of Leicester NHS Trust (ref 11444) on 29 September 2015. Therefore, all steps have been taken when designing this protocol to minimise all ethical implications and ensure patient welfare. Two substantial amendments were made to this protocol, approved on 03 December 2015 and 01 August 2016 to add saliva collection and fatigue questionnaires and muscle $\mathrm{O}_{2}$ saturation, respectively. The protocol presented here represents the most up-to-date version.

\section{Dissemination plan}

The results are planned for publication in early 2018. We plan to present the data at relevant national and international conferences, as well as publish the findings in relevant journals. Participant level data will be available at a later date.

\section{Trial registration}

This study is registered with the ISRCTN (ISRCTN38935454). The registration was completed after recruitment of the first patient, so the study was registered retrospectively.

Contributors PJH wrote this manuscript; JN was involved in study design and protocol preparation; TJW was involved in study design and preparation of protocol amendments; NCB was involved in study design; ACS lead study design and protocol preparation. All authors reviewed this manuscript.

Funding We are grateful to the Stoneygate Trust for partially funding this study. This work is supported by the National Institute for Health Research (NIHR) Diet, Lifestyle \& Physical Activity Biomedical Research Unit based at University Hospitals of Leicester and Loughborough University. The views expressed are those of the authors and not necessarily those of the NHS, the NIHR or the Department of Health. Competing interests None declared.

Patient consent Obtained from patients.

Ethics approval REC East Midlands-Derby (ref 15/EM/0391).

Provenance and peer review Not commissioned; externally peer reviewed.

Open Access This is an Open Access article distributed in accordance with the terms of the Creative Commons Attribution (CC BY 4.0) license, which permits others to distribute, remix, adapt and build upon this work, for commercial use, provided the original work is properly cited. See: http://creativecommons.org/ licenses/by/4.0/

(c) Article author(s) (or their employer(s) unless otherwise stated in the text of the article) 2017. All rights reserved. No commercial use is permitted unless otherwise expressly granted.

\section{REFERENCES}

1. Shlipak MG, Fried LF, Cushman M, et al. Cardiovascular mortality risk in chronic kidney disease: comparison of traditional and novel risk factors. JAMA 2005;293:1737-45.

2. Caskey F, Castledine C, a D, et al. UK Renal Registry 2015 18th Annual Report of the Renal Association. Nephron 2016;132(suppl1):1-366 https://www.renalreg.org/.

3. Johansen KL, Shubert T, Doyle J, et al. Muscle atrophy in patients receiving hemodialysis: effects on muscle strength, muscle quality, and physical function. Kidney Int 2003;63:291-7 http://www. sciencedirect.com/science/article/pii/S0085253815488714.

4. Fried LF, Lee JS, Shlipak M, et al. Chronic kidney disease and functional limitation in older people: health, aging and body composition study. J Am Geriatr Soc 2006;54:750-6.

5. Fouque D, Kalantar-Zadeh K, Kopple J, et al. A proposed nomenclature and diagnostic criteria for protein-energy wasting in acute and chronic kidney disease. Kidney Int 2008;73:391-8.

6. Moreau K, Desseix A, Germain C, et al. Body composition in 98 patients awaiting kidney transplantation. Nutrition 2014;30:186-91.

7. Johnson CP, Gallagher-Lepak S, Zhu YR, et al. Factors influencing weight gain after renal transplantation. Transplantation 1993;56:822-6.

8. Baum CL. Weight gain and cardiovascular risk after organ transplantation. JPEN J Parenter Enteral Nutr 2001;25:114-9 http:// www.thehastingscenter.org/Publications/BriefingBook/Detail.aspx? $\mathrm{id}=2198$.

9. van den Ham EC, Kooman JP, Christiaans $\mathrm{MH}$, et al. Posttransplantation weight gain is predominantly due to an increase in body fat mass. Transplantation 2000;70:241-2. 
10. Oberg BP, McMenamin E, Lucas FL, et al. Increased prevalence of oxidant stress and inflammation in patients with moderate to severe chronic kidney disease. Kidney Int 2004;65:1009-16.

11. Molnar MZ, Carrero JJ, Mucsi I, et al. Comparison of the malnutrition-inflammation score in chronic kidney disease patients and kidney transplant recipients. Int Urol Nephrol 2015;47:1025-33.

12. Stenvinkel $P$, Heimbürger $O$, Paultre $F$, et al. Strong association between malnutrition, inflammation, and atherosclerosis in chronic renal failure. Kidney Int 1999;55:1899-911.

13. Carrero JJ, Stenvinkel P, Cuppari L, et al. Etiology of the proteinenergy wasting syndrome in chronic kidney disease: a consensus statement from the International Society of Renal Nutrition and Metabolism (ISRNM). J Ren Nutr 2013;23:77-90.

14. Hauser AB, Stinghen AE, Kato $S$, et al. Characteristics and causes of immune dysfunction related to Uremia and Dialysis. Perit Dial Int 2008;28(Suppl 3):183-7

15. Girndt M, Sester U, Sester M, et al. Impaired cellular immune function in patients with end-stage renal failure. Nephrol Dial Transplant 1999;14:2807-10.

16. Hutchinson P, Chadban SJ, Atkins RC, et al. Laboratory assessment of immune function in renal transplant patients. Nephrol Dial Transplant 2003;18:983-9 http://ndt.oxfordjournals. org/cgi/doi/.

17. Vereyken EJ, Kraaij MD, Baan CC, et al. A shift towards proinflammatory CD16+ monocyte subsets with preserved cytokine production potential after kidney transplantation. PLoS One 2013;8:e70152 http://journals.plos.org/plosone/article?id=.

18. Lu GY, Xu RJ, Zhang SH, et al. Alteration of circulatory platelet microparticles and endothelial microparticles in patients with chronic kidney disease. Int J Clin Exp Med 2015;8:16704-8.

19. VanWijk MJ, VanBavel E, Sturk A, et al. Microparticles in cardiovascular diseases. Cardiovasc Res 2003;59:277-87.

20. Stehr MD, von Lengerke T. Preventing weight gain through exercise and physical activity in the elderly: a systematic review. Maturitas 2012;72:13-22.

21. Peterson MD, Sen A, Gordon PM. Influence of resistance exercise on lean body mass in aging adults: a meta-analysis. Med Sci Sports Exerc 2011;43:249-58.

22. de Vries NM, van Ravensberg CD, Hobbelen JS, et al. Effects of physical exercise therapy on mobility, physical functioning, physical activity and quality of life in community-dwelling older adults with impaired mobility, physical disability and/or multi-morbidity: a metaanalysis. Ageing Res Rev 2012;11:136-49.

23. Chou CH, Hwang CL, Wu YT. Effect of exercise on physical function, daily living activities, and quality of life in the frail older adults: a meta-analysis. Arch Phys Med Rehabil 2012;93:237-44.

24. Woods JA, Vieira VJ, Keylock KT, a WJ, Exercise KKT. Exercise, inflammation, and innate immunity. Immunol Allergy Clin North Am 2009;29:381-93.

25. Gleeson M, Bishop NC, Stensel DJ, et al. The anti-inflammatory effects of exercise: mechanisms and implications for the prevention and treatment of disease. Nat Rev Immunol 2011;11:607-15 http:// www.nature.com/doifinder/.

26. Nieman DC, Pedersen BK. Exercise and immune function. Sports Medicine 1999;27:73-80.

27. Walsh NP, Gleeson M, Shephard RJ, et al. Position statement. Part one: immune function and exercise. Exerc Immunol Rev 2011;17:6-63.

28. Babbitt DM, Diaz KM, Feairheller DL, et al. Endothelial activation microparticles and inflammation status improve with exercise training in african americans. Int J Hypertens 2013;2013:1-8 http://www. pubmedcentral.nih.gov/articlerender.fcgi?artid=3652180\&tool= pmcentrez\&rendertype=abstract.

29. Wahl P, Jansen F, Achtzehn S, et al. Effects of high intensity training and high volume training on endothelial microparticles and angiogenic growth factors. PLoS One 2014;9:e96024 http://dx.plos. org/.

30. Castaneda C, Layne JE, Munoz-Orians L, et al. A randomized controlled trial of resistance exercise training to improve glycemic control in older adults with type 2 diabetes. Diabetes Care 2002;25:2335-41.

31. Mercer TH, Crawford C, Gleeson NP, et al. Low-volume exercise rehabilitation improves functional capacity and self-reported functional status of Dialysis patients. Am J Phys Med Rehabil 2002;81:162-7.
32. Koufaki P, Mercer TH, Naish PF. Effects of exercise training on aerobic and functional capacity of end-stage renal disease patients. Clin Physiol Funct Imaging 2002;22:115-24.

33. Mustata S, Groeneveld S, Davidson W, et al. Effects of exercise training on physical impairment, arterial stiffness and health-related quality of life in patients with chronic kidney disease: a pilot study. Int Urol Nephrol 2011;43:1133-41.

34. Viana JL, Kosmadakis GC, Watson EL, et al. Evidence for antiinflammatory effects of exercise in CKD. J Am Soc Nephrol 2014;25:2121-30 http://www.jasn.org/cgi/doi/.

35. Van Craenenbroeck AH, Van Ackeren K, Hoymans VY, et al. Acute exercise-induced response of monocyte subtypes in chronic heart and renal failure. Mediators Inflamm 2014;2014:1-11.

36. Johansen KL. Exercise and chronic kidney disease: current recommendations. Sports Med 2005;35:485-99.

37. Singh SJ, Morgan MD, Scott S, et al. Development of a shuttle walking test of disability in patients with chronic airways obstruction. Thorax 1992;47:1019-24.

38. Revill SM, Morgan MDL, Singh SJ, et al. The endurance shuttle walk: a new field test for the assessment of endurance capacity in chronic obstructive pulmonary disease. Thorax 2007;1999:213-22.

39. Greenwood SA, Lindup H, Taylor K, et al. Evaluation of a pragmatic exercise rehabilitation programme in chronic kidney disease. Nephrol Dial Transplant 2012;27(Suppl 3):iii126-34.

40. Wilund KR, Tomayko EJ, Wu PT, P-t W, et al. Intradialytic exercise training reduces oxidative stress and epicardial fat: a pilot study. Nephrol Dial Transplant 2010;25:2695-701 http://ndt.oxfordjournals. org/cgi/doi/.

41. Painter $P$, Marcus RL. Assessing physical function and physical activity in patients with CKD. Clin J Am Soc Nephrol 2013;8:861-72.

42. van der Heyde HC, Gramaglia I, Combes V, et al. Flow cytometric analysis of microparticles. Methods Mol Biol 2011;699:337-54.

43. Ziegler-Heitbrock L, Hofer TPJ. Toward a refined definition of monocyte subsets. Front Immunol 2013:1-5 http://journal.frontiersin. org/article/10.3389/fimmu.2013.00023/full.

44. Nielsen MH, Beck-Nielsen $\mathrm{H}$, Andersen MN, et al. A flow cytometric method for characterization of circulating cell-derived microparticles in plasma. J Extracell Vesicles 2014;3:1-12 http:// www.pubmedcentral.nih.gov/articlerender.fcgi?artid=3916676\&tool= pmcentrez\&rendertype $=$ abstract

45. Jy W, Horstman LL, Jimenez JJ, et al. Measuring circulating cellderived microparticles. J Thromb Haemost 2004;2:1842-3.

46. Eston R, Reilly T. Kinanthropometry and exercise physiology laboratory manual: anthropometry, 2009.

47. Chertow GM, Lowrie EG, Wilmore DW, et al. Nutritional assessment with bioelectrical impedance analysis in maintenance hemodialysis patients. J Am Soc Nephrol 1995;6:75-81.

48. Squara $P$, Denjean D, Estagnasie $P$, et al. Noninvasive cardiac output monitoring (NICOM): a clinical validation. Intensive Care Med 2007;33:1191-4.

49. Obrador GT, Pereira BJ. Anaemia of chronic kidney disease: an under-recognized and under-treated problem. Nephrol Dial Transplant 2002:17(Suppl 11):44-6.

50. Cheung WW, Paik KH, Mak RH. Inflammation and cachexia in chronic kidney disease. Pediatr Nephrol 2010;25:711-24 http://link. springer.com/.

51. Chao CT, Huang JW, Chiang CK. COGENT (COhort of GEriatric Nephrology in NTUH) study group. Functional assessment of chronic illness therapy-the fatigue scale exhibits stronger associations with clinical parameters in chronic dialysis patients compared to other fatigue-assessing instruments. Peer J 2016;4:e1818.

52. Jhamb M, Liang K, Yabes J, Steel L, et al. Prevalence and correlates of fatigue in chronic kidney disease and end-stage renal disease: are sleep disorders a key to understanding fatigue? Am J Nephrol 2013;38:489-95

53. Gardner AW, Parker DE, Webb N, et al. Calf muscle hemoglobin oxygen saturation characteristics and exercise performance in patients with intermittent claudication. J Vasc Surg 2008;48:644-9.

54. Wüst RC, Degens $H$. Factors contributing to muscle wasting and dysfunction in COPD patients. Int J Chron Obstruct Pulmon Dis 2007;2:289-300.

55. Grassi B, Quaresima V. Near-infrared spectroscopy and skeletal muscle oxidative function in vivo in health and disease: a review from an exercise physiology perspective. J Biomed Opt 2016;21:091313 http://biomedicaloptics.spiedigitallibrary.org/article.aspx? 